\title{
Magnetic behaviour of narrow track thin-film heads
}

\author{
W. F. Druyvesteyn, E. L. M. Raemaekers, R. D. J. Verhaar, and J. de Wilde \\ Philips Research Laboratories, 5600 MD Eindhoven, The Netherlands
}

\section{J. H. J. Fluitman and J. P. J. Groenland}

Twente University of Technology, Enschede, The Netherlands

\begin{abstract}
The influence of the trackwidth on the performance of thin film heads has been tested. Results of experiments on the wafer have indicated an increase in the head efficiency with decreasing trackwidth. This was underlined by measurements of the head fringe field and tape recording experiments. A model which takes the domain structure into account has been developed to interpret this behaviour.
\end{abstract}

PACS numbers: 75.60.Ch, 85.70. - w

\section{INTRODUCTION}

Thin film heads are especially suited for use in recording systems with a high information density e.g. a narrow trackwidth. Therefore it is important to know the influence of the trackwidth $w$ on its recording performance. The magnetic yoke of a thin film head has dimensions such that it will not be magnetised uniformly but will split up into domains, owing to the high demagnetising field. A study of this domain structure revealed [1] that it greatly depends on the trackwidth of the head.

(a) Physical aspects of this trackwidth dependence.

It is usual to have the easy axis (e.a.) of magnetisation paraliel to the turn (fig.1). In this situation domains will occur with a period $d$ which depends on $w[6]$.

When a magnetic field $\mathrm{H}$ is applied perpendicular to the turn, domain wall displacement and rotation of the magnetisation will occur as illustrated in fig. 3. We assume that the coercive field is equal to zero so that domain wall displacement occurs at the lowest fields.

If $w>d$ domain wall displacement hardly contributes to the magnetisation in the direction of the field. In this case only rotation plays a role so that the permeability is determined by the ansiotropy field $H_{K}, \mu_{0} \mu_{r}=B_{s} / H_{K}$, where $B_{s}$ is equal to the magnetic induction of the saturated strip. However, if $w$ is reduced, wall displacement contributes to the permeability and $\mu_{0} \mu_{\mathrm{r}}>\mathrm{B}_{\mathrm{s}} / \mathrm{H}_{\mathrm{K}}$.

If the e.a. is perpendicular to the turn only wall displacement governs the magnetisation process. Therefore no trackwidth dependence will be expected.

(b) Relation between efficiency and permeability.

For a thin film head the relation between the efficiency $\eta$ and the permeability of a thin film head has been derived by Paton [2]. In general $\eta$ increases with increasing $\mu_{\mathrm{r}}$, but in some heads the influence is very weak. In Table I we give some examples of heads with different poletip length $p$ and height $h$.

Table I clearly shows that for some heads the influence of $\mu_{r}$ on $\eta$ is quite strong (type I) while for other heads (type II) hardly an influence exists. In our experiments type I was chosen as we wanted to study the influence of the domain structure on $\eta$. However, in a recording system one mostly prefers to eliminate this effect and uses type II head.

TABLE I

\begin{tabular}{|c|c|c|c|c|c|c|}
\hline & $\mu$ & 500 & 800 & 1000 & 1500 & 2000 \\
\hline 1 & $\begin{array}{l}\mathrm{p}=1 \mu \mathrm{m} \\
\mathrm{g}=2 \mu \mathrm{m} \\
\mathrm{h}=20 \mu \mathrm{m}\end{array}$ & 47 & 56 & 60 & 67 & 72 \\
\hline II & $\begin{array}{l}\mathrm{p}=4 \mu \mathrm{m} \\
\mathrm{g}=2 \mu \mathrm{m} \\
\mathrm{h}=5 \mu \mathrm{m}\end{array}$ & 87 & 91 & 93 & 95 & 97 \\
\hline
\end{tabular}

Efficiency $\eta$ (in \%) as a function of $\mu_{\mathrm{r}}$ for two types of heads following [2]

\section{MANUFACTURING OF THE HEADS}

In order to measure the influence of $w$ on the magnetic behaviour of a thin film head, special test circuits have been designed containing a series of identical single turn heads with different $w$. Heads have been processed with a symmetrical (two Permalloy shields) structure on oxydised silic on wafers. Non-magnetostrictive Permalloy films with a thickness of $1 \mu \mathrm{m}$ are obtained by electrodeposition. A $0.06 \mu \mathrm{m}$ thick sputtered $\mathrm{NiFe}$ layer serves as the plating base. Some typical magnetic parameters of this electrodeposited material are: $H_{c}=80 \mathrm{~A} / \mathrm{m} ; H_{K}=400 \mathrm{~A} / \mathrm{m}$, while most films are made with a welldefined e.a.. From these layers the heads are formed by means of chemical etching or ion-milling. Sputtered $\mathrm{SiO}_{2}$ is used as an insulator and sputtered MoAuMo as a conductor.

The heads are encapsulated and polished so that either the height $\mathrm{h}=\mathbf{2 0} \mu \mathrm{m}$ (type I) or $\mathrm{h}=5 \mu \mathrm{m}$ (type II).

\section{WAFER TESTING}

The magnetic properties of the heads with $w$ ranging from 600 $\mu \mathrm{m}$ to $10 \mu \mathrm{m}$ have been measured on the wafer.

In order to get a clear picture of the influence of $w$, it is necessary to measure a large number of identical heads. Therefore the electronic setup was arranged around an Electroglas 900 wafer tester. The induced voltage $v$ across the test turn (fig. 1) was measured as a function of the ac current $i$ flowing in the writing turn. The frequency was $0.5 \mathrm{MHz}$. The effective permeability $\mu_{r}$ was found from the mutual inductance $M$ by using the transmission line model [2].

In the heads with e.a. oriented parallel to the writing turn, all experiments indicate that $\mu_{\mathrm{r}}$ increases with decreasing trackwidth. This is illustrated in fig. 1. Curve I represents the results of heads formed by means of chemical etching and curve $1 /$ heads formed by ionmilling. No difference can be seen. If $w$ is larger than $200 \mu \mathrm{m}$, only a slight increase can be detected. However, if $w<50 \mu \mathrm{m}$, especially in the circuits with w down to $10 \mu \mathrm{m}$, we see a dramatic increase in $\mu_{\mathrm{r}}$ (fig. 1 , curve (II).

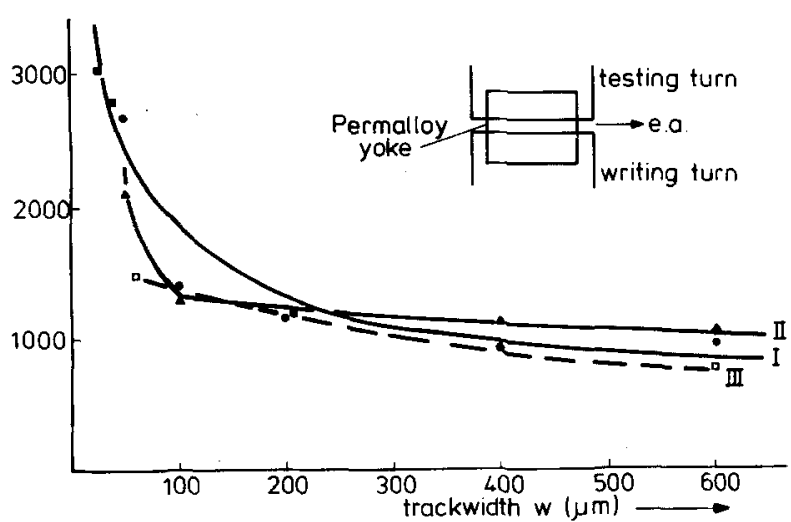

Fig. 1. The trackwidth dependence of $\mu_{\mathrm{r}}$ as measured in wafer testing experiments. 
On the other hand the heads with the e.a. perpendicular to the turn do not show this behaviour, but taking all heads on one wafer into account the standard deviation is very high.

The $\mu_{\mathrm{r}}$ of these heads depends on the accidental occurrence of pinning points and structural imperfections of the Permalloy which was in agreement with the observed domain structures.

\section{HEAD FRINGE FIELD}

The head fringe field has been measured with a magnetoresistive transducer (MRT) and analysed in a way described earlier [3].

The fields on the energised heads were measured by placing them underneath the magnetoresistor at an accurately adjusted distance, and moving them laterally while keeping the separation at a constant magnitude. The thickness of the MRT was $0.03 \mu \mathrm{m}$ and its height $2.8 \mu \mathrm{m}$. Since the latter value is large compared with the field inhomogeneities, the analysis of the field tracks takes the transducer response into account [4]. In this way 'field tracks' were produced as a function of head/transducer separation and as a function of writing current. The orientation of the transducer is such that the perpendicular field component is detected. Measurements were performed on heads with a gap length of $2.6 \mu \mathrm{m}$ and pole tips of 1.15 and $0.9 \mu \mathrm{m}$ respectively.

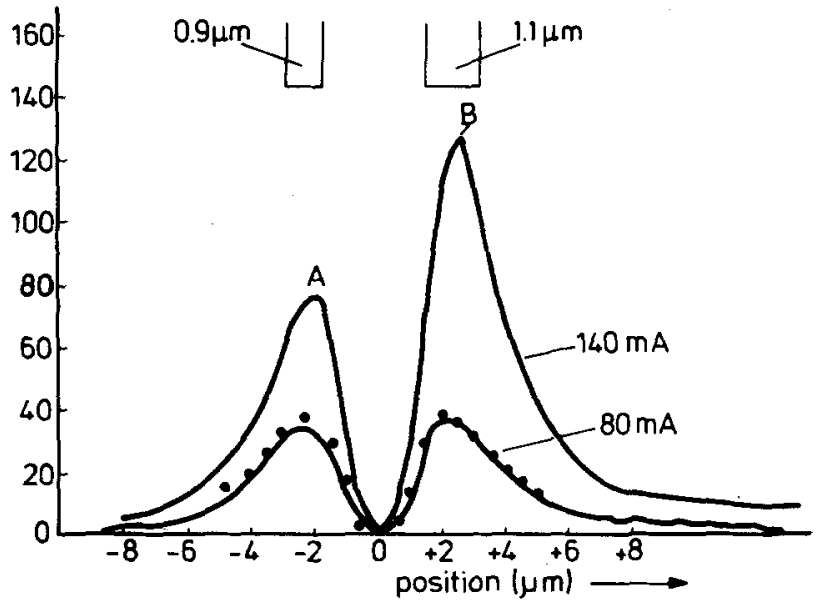

Fig. 2. Typical sensor output for head fringe measurements at a head/transducer distance of $1.0 \mu \mathrm{m}$. The solid curve is experimental, while the dots represent computed results. In the curve at $I=140 \mathrm{~mA}$ the thinnest Permalloy shield is saturated (peak A).

Occasionally these calculations must include the field components introduced by the transducer mirror image in the pole tips.

The relation between the writing current and the head field was found to be linear up to $100 \mathrm{~mA}$. However, a small hysteretic effect was found indicating the presence of domains. At $100 \mathrm{~mA}$ saturation of the thinnest poletip starts. The current dependence of the peak amplitudes is illustrated in fig. 2. (Note that the response of the MRT is quadratic.)

The structure of the head field has been analysed by comparing the experimental numbers with the simulated results. The form of the theoretical field was calculated by means of the method of van Lier [5].

The curves were fitted by using the head efficiency as a parameter. The agreement between theoretical and experimental curves is good (cf. fig. 2).

TABLE |f

\begin{tabular}{|c|cccc|}
\hline & & & \multicolumn{2}{c|}{ recording } \\
$w(\mu \mathrm{m})$ & wafer & field & $12 \mathrm{kHz}$ & $18 \mathrm{kHz}$ \\
\hline 100 & 62 & 63 & 59 & 55 \\
200 & 55 & 55 & 59 & 51 \\
400 & 45 & 45 & 46 & 49 \\
600 & 45 & 45 & 45 & 45 \\
\hline
\end{tabular}

Head efficiency (in \%) from different experiments.

\section{RECORDING EXPERIMENTS}

Recording experiments on tape have been carried out with the same heads as used in the field measurements.

The reproducing efficiency was measured on video tape $\left(H_{c}=55 \times 10^{3} \mathrm{~A} / \mathrm{m}\right)$. A harmonic signal was recorded with a ferrite head. The tape velocity was $19 \mathrm{~cm} / \mathrm{s}$ and the recorded frequencies were $12 \mathrm{kHz}$ and $18 \mathrm{kHz}$ respectively. These signals were reproduced by means of the thin film heads. The detected levels were compared with the signal from a conventional ferrite head with known efficiency.

The resulting efficiency $\eta$ has a function of the trackwidth of the head as been given in Table II. It is clear that all numbers in this table show the same increase with decreasing trackwidth.

We tried to do writing experiments with the same heads, although the low efficiency and the thin Permalloy yoke (saturation) limited the head field. Therefore audio tape $\left(H_{c}=26 \times 10^{3} \mathrm{~A} / \mathrm{m}\right)$ was chosen. The written information was reproduced with a ferrite head. Again the same track dependence was found: the non-linear behaviour of the recording process even emphasised this effect.

The same recording experiments have been performed with type II heads (cf. Table I).

Indeed when $w$ was varied between $50 \mu \mathrm{m}$ and $600 \mu \mathrm{m}$ the head efficiency was constant both in the writing and reading measurements.

\section{CALCULATIONS}

A model has been developed for calculating the influence of $w$ on $\mu_{\mathrm{r}}$. To characterise the magnetic behaviour of the head material, we consider it as an infinite strip with width $w$ placed in an external magnetic field parallel to this strip (fig. 3). In the yoke of a thin film head, flux circulates in such a way that the direction of the magnetisation in the upper and lower shield are opposite to each other and are closed through the air gap and by the magnetic material in the back of the head. A small amount of the total flux will be closed outside the head (head fringe field) but for all the heads we used it can be shown that this flux is negligible compared with the total flux running in the head. Therefore the demagnetising field at the front of the head will be very sinall.

If the easy axis of the magnetisation is perpendicular to the strip, domains will occur with a period d depending on $w$ [6].

The permeability is calculated by minimising the total magnetic energy with respect to the rotation angle $\alpha$ and the geometry of the closure domains e.g. $\ell_{1}$ (or $\ell_{2}$ ). Three energy terms are considered: the anisotropy energy, the wall energy and the magnetic field energy.

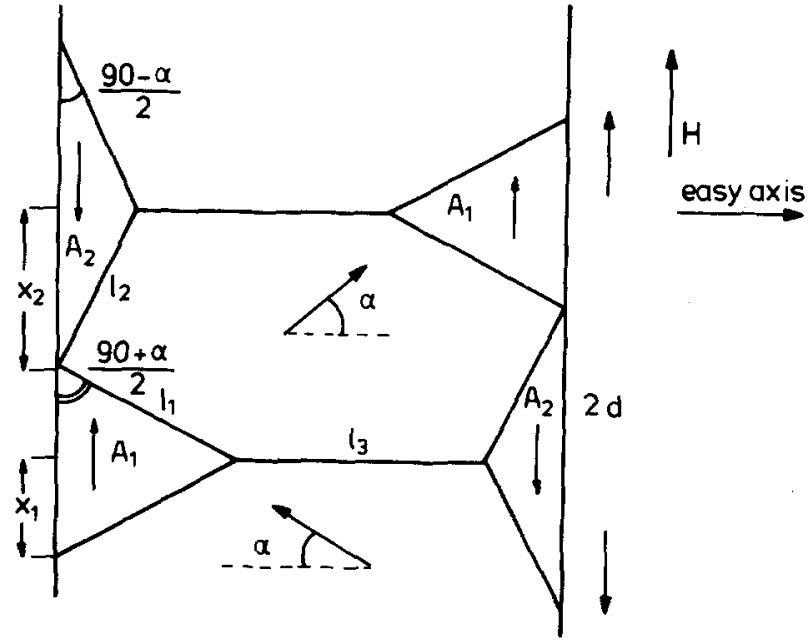

Fig. 3. Domain configurations as used in the calculations. 


\section{CONCLUSIONS}

$E^{\prime}=\frac{K d}{2 t}\left[\left(\ell_{1}{ }^{2}+\ell_{2}{ }^{2}\right) \cos \alpha+\left(2 w d-\ell_{1}{ }^{2} \cos \alpha-\ell_{2}{ }^{2} \cos \alpha\right) \sin ^{2} \alpha\right]$

$+\frac{\gamma d}{t}\left[2 l_{1} \sin ^{2}\left(45-\frac{\alpha}{2}\right)+2 l_{2} \sin ^{2}\left(45+\frac{\alpha}{2}\right)+\left[w-l_{1} \sin \left(45+\frac{\alpha}{2}\right)\right.\right.$

$\left.\left.-\ell_{2} \sin \left(45-\frac{\alpha}{2}\right)\right] \cos ^{2} \alpha\right]+\frac{M_{s} H d}{t}\left[-\left(\omega d-\frac{\ell_{1}{ }^{2}+l_{2}{ }^{2}}{2} \cos \alpha\right) \times\right.$

$\left.\sin \alpha-1 / 2 \ell_{1}^{2} \cos \alpha+1 / 2 \ell_{2}^{2} \cos \alpha\right]$

where $t$ is the thickness of the films, $K$ the uniaxial anisotropy constant, $\gamma$ the wall energy of an $180^{\circ}$ Bioch wall, $M_{s}$ the saturation magnetisation of the strip.

When applying a field $H$, the number of domains remains constant, i.e. we assume that this $d$ is fixed:

$$
d=\sqrt{\frac{\gamma w}{K}}=\ell_{1} \cos \left(45+\frac{\alpha}{2}\right)+l_{2} \cos \left(45-\frac{\alpha}{2}\right)
$$

The energy thus depends on two variables, e.g. $\alpha$ and $\ell_{1}$. For a stable domain configuration the first derivations of $E^{\prime}$ with respect to $\alpha$ and $l_{1}$ should be zero. Solving $\alpha$ and $l_{1}$ from these equations is straightforward, therefore we shall not reproduce this here [7]. The results in terms of $M / M_{s}$ as a function of $H$ for different values of $w$ are given in fig. 4. In this model calculation the parameters were: $H_{K}=400 \mathrm{~A} / \mathrm{m}$, $\gamma=2 \times 10^{-3} \mathrm{Jm}^{-2}, \mathrm{~K}=2 \mathrm{Jm}^{-3}$ and the trackwidth varied between 40 $\mu \mathrm{m}$ and $100,000 \mu \mathrm{m}$.

The relative permeability deduced from these curves increases with decreasing trackwidth, even in this simple model.

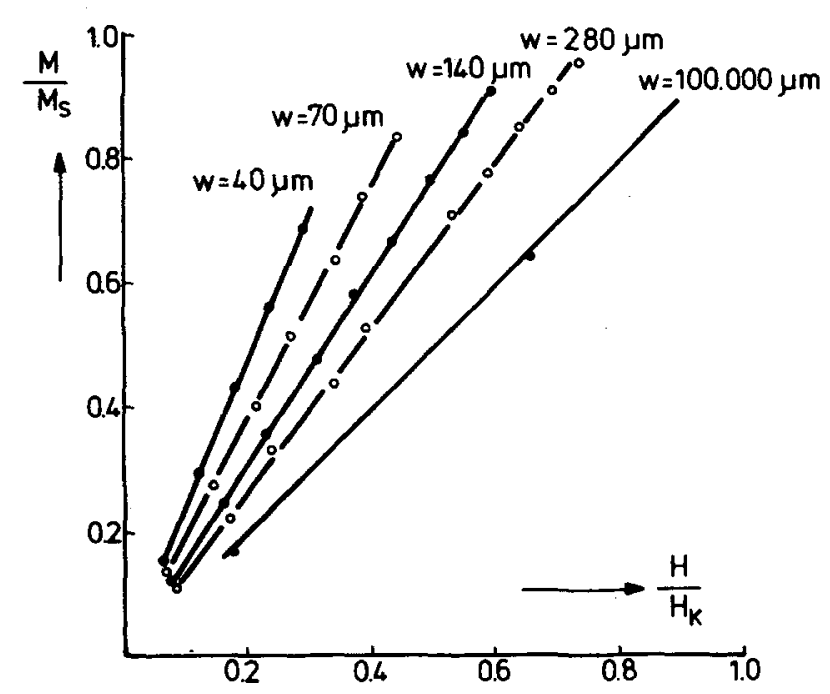

Fig. 4. The almost linear relation between $M$ and $H$. All points refer to numerical results. (n)
In the theoretical analysis [2] of the magnetic behaviour of thin film heads homogeneous magnetisation is assumed whereas the relation between $B$ and $H$ is characterised by the material constant $\mu_{r}$. However, it is known that the NiFe yoke of the head is not uniformly magnetised but is split up into domains. We have shown that nevertheless several experimental results can be described with $\mu_{\mathrm{r}}$ as an adjustable parameter. The permeability depends on the trackwidth of the head, if the easy axis of the magnetisation is parallel to the turn (fig. 1). From wafer testing experiments we conclude that the efficiency of a narrow track head should increase with decreasing trackwidth. Head fringe field measurements and tape recording experiments are in agreement with this fact and have shown the reliability of wafer test measurements.

The fringe field of the head is in agreement with the model calculations of Van Lier [5]. The dead layer in the Permalloy yoke, if present, is within the experimental error of $0.1 \mu \mathrm{m}$. The efficiency values of the heads achieved in the several experiments are in reasonable agreement with each other (Table II), and the model calculation which takes the domain structure into account, shows the same increase in the $\mu_{\mathrm{r}}$ with decreasing trackwidth.

\section{REFERENCES}

1. W.F. Druyvesteyn, L. Postma, G. Somers, IEEE Trans. on Magn., MAG-15, 1613 (1979).

2. A. Paton, J. of Appl. Phys. 42, 5868 (1971).

3. J.H.J. Fluitman, J.P.J. Groenland, IEEE Trans. on Magn., MAG-15. 1634 (1979).

4. J.H.J. Fluitman, IEEE Trans. on Magn., MAG-14, 433 (1978).

5. J.C. van Lier, Conf. on Video and Data Recording, PROC. IRE 26, 285 (1973).

6. S. Middelhoek, Thesis Amsterdam 1961.

7. Details about the calculations will be supplied on request.

(1)

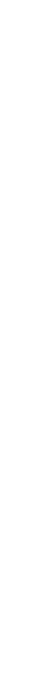

